\title{
Positive nin one binding protein expression predicts poor outcome in prostate cancer
}

\author{
JIE CHEN* ${ }^{*}$, JUNKAI WANG* ${ }^{*}$ XINGANG CUI, YUSHAN LIU, LEI YIN, \\ YAO LI, LU CHEN, DANFENG XU and YI GAO \\ Department of Urology, Affiliated Changzheng Hospital of the Second Military Medical University, \\ Shanghai 200003, P.R. China
}

Received December 3, 2013; Accepted May 2, 2014

DOI: $10.3892 / \mathrm{mmr} .2014 .3066$

\begin{abstract}
Nin one binding protein (NOB) 1 has been reported to be involved in cancer. However, the role of NOB1 in prostate cancer (PCa) has yet to be elucidated. The present study aimed to investigate the clinical role of NOB1 in PCa. The gene and protein expression levels of NOB1 were detected using quantitative polymerase chain reaction and western blot analysis in fresh samples of PCa and matched adjacent noncancerous tissues $(n=32)$. NOB1 expression was also assessed using immunohistochemistry in a large number of patients with $\mathrm{PCa}(\mathrm{n}=456)$ and paired adjacent noncancerous tissues between December 2003 and December 2010. The association between NOB1 expression and clinicopathological features and prognosis was investigated. The expression of NOB1 was found to be higher in the fresh samples of PCa than in the paired adjacent noncancerous tissues at the gene and protein levels. Immunohistochemistry revealed that the positive expression rates of NOB1 in PCa and the paired adjacent noncancerous tissues were 53\% (242/456) and 6.1\% (28/456), respectively. NOB1 expression was significantly correlated with Gleason score $(\mathrm{P}<0.001)$ and distant metastasis $(\mathrm{P}<0.001)$. Furthermore, patients with PCa with positive NOB1 expression had lower overall survival and recurrence-free survival (RFS) compared with those with negative NOB1 expression. Multivariate analysis showed that NOB1 was an independent marker of RFS (hazard ratio, 3.45; 95\% confidence interval, 1.41-6.35; $\mathrm{P}=0.006)$. These findings suggest that NOB1 may be a potential prognostic indicator for $\mathrm{PCa}$.
\end{abstract}

Correspondence to: Dr Yi Gao, Department of Urology, Affiliated Changzheng Hospital of the Second Military Medical University, 415 Fengyang Road, Shanghai 200003, P.R. China

E-mail: gaoyishanghai@163.com

*Contributed equally

Key words: prostate cancer, nin one binding protein 1, prognosis, biomarker

\section{Introduction}

Prostate cancer (PCa) is an aggressive invasive tumor, which is one of the most prevalent malignancies in males and the second most common cause of male cancer-related mortality (1). PCa is a clinically heterogeneous-multifocal disease and its incidence is increasing (2). The mechanisms influencing the progression and prognosis of PCa involve a multi-step process (3). Novel effective diagnostic and prognostic biomarkers for PCa are required to prevent the overtreatment of indolent tumors and to ensure early detection of aggressive $\mathrm{PCa}$, which requires early treatment intervention (4).

Nin one binding protein (NOB1), encoded by the NOB1 gene, is a subunit of the $26 \mathrm{~S}$ proteasome and has a key role in the protein degradation pathway (5). The NOB1 protein is composed of a PIN domain and a $\mathrm{C}$ terminal zinc ribbon domain (6), which has been reported to function as a transcriptional regulator. NOB1 may have a role in cell cycle progression, drug resistance and oncogenesis $(7,8)$. A previous study showed that knockdown of NOB1 expression inhibits cell proliferation and migration in human gliomas, suggesting that NOB1 promotes glioma cell growth and migration, and may be a candidate for molecular targeting (9). These findings indicate that NOB1 may be involved in various types of tumors and may serve as an oncogenic factor. However, the expression of NOB1 and its significance in $\mathrm{PCa}$ remains unclear. The present study aimed to investigate NOB1 expression to assess its prognostic significance in patients with PCa with long-term follow-up data and its association with clinicopathological features.

\section{Materials and methods}

Patient and sample tissues. Fresh PCa samples and paired adjacent noncancerous tissues $(n=32)$ were obtained from Changzheng Hospital (Shanghai, China) for quantitative polymerase chain reaction (qPCR) and western blot analysis of NOB1 gene and protein expression, respectively. Furthermore, the surgical prostate cancer database (http://ef.inbi.ras.ru) was used to retrospectively analyze 456 patients with $\mathrm{PCa}$. All of the patients used in the present study had undergone prostatectomy, transrectal prostate biopsy under ultrasound guidance or transurethral resection of the prostate between 
December 2003 and December 2010. All samples were fixed with formalin and embedded in paraffin.

Clinical data, including the Gleason score, baseline prostate-specific antigen (PSA) level, PSA nadir, level of distant metastasis and follow-up status, were retrospectively obtained from the Changzheng Hospital medical records. Paraffin-embedded prostate tissues were obtained from the Department of Urology, Affiliated Changzheng Hospital of the Second Military Medical University (Shanghai, China). Serum PSA-based biochemical recurrence and PCa-specific death were used as the end-points (10).

The present study was approved by the Review Board of the Second Military Medical University (Shanghai, China) and was performed in accordance with the Declaration of Helsinki. Signed informed consent was obtained from all patients prior to surgery.

qPCR analysis. Total RNA was extracted from fresh $\mathrm{PCa}$ samples using an RNA extraction kit according to the manufacturer's instructions and reverse transcribed using a Reverse Transcription kit (Qiagen, Valencia, CA, USA). Complementary (c)DNA (6 ng) SYBR ${ }^{\circledR}$ Green (Invitrogen Life Technologies, Carlsbad, CA, USA) qPCR analysis was then performed. The primers used were as follows: Forward: 5'-AAGTGAGGAGGAGGAGGAG-3' and reverse: 5'-ACTTTCTTCAGGGTCTTGTTC-3' for NOB1; and forward: 5'-GTGGACATCCGCAAAGAC-3' and reverse: 5'-AAAGGGTGTAACGCAACTA-3' for $\beta$-actin. Data were analyzed using the $\Delta \Delta \mathrm{Ct}$ method and were normalized using $\beta$-actin mRNA expression.

Western blot analysis. Fresh PCa samples were lysed in cell lysis buffer (Cell Signaling Technology, Inc., Beverly, MA, USA) and quantified using a Bicinchoninic Acid Assay kit (Sigma-Aldrich, St Louis, MO, USA). Proteins were separated using 10\% SDS-PAGE, then electroblotted onto polyvinylidene fluoride membranes (Millipore Corporation, Billerica, MA, USA). Membranes were immunoblotted overnight at $4^{\circ} \mathrm{C}$ with primary antibodies against human NOB1 (dilution, 1:2,000; LifeSpan Biosciences Inc., Seattle, WA, USA) and $\beta$-actin (dilution, 1:5,000; LifeSpan Biosciences Inc.). Following washing with Tris-buffered saline containing Tween-20 three times, membranes were incubated with horseradish peroxidase (HRP)-conjugated secondary antibody immunoglobulin G (dilution, 1:2,000; Santa Cruz Biotechnology Inc., Santa Cruz, CA, USA) for $2 \mathrm{~h}$. Immunoreactive bands were detected using an enhanced chemiluminescence detection reagent (Pierce Biotechnology, Inc., Rockford, IL, USA). Images were analyzed using Quantity One ${ }^{\circledR}$ software (Bio-Rad, Hercules, CA, USA). All experiments were performed in triplicate.

Immunohistochemical analysis. All slides were assessed by two blinded pathologists and a consensus was reached. Sections were deparaffinized, hydrated and immersed in peroxidase-blocking solution (Qiagen) to inhibit any endogenous peroxidase activity. For antigen retrieval, microwave pretreatment was performed using $0.01 \mathrm{~mol} / 1$ citrate buffer (pH 6.0) for $30 \mathrm{~min}$. Sections were incubated overnight at $4^{\circ} \mathrm{C}$ with mouse monoclonal antibodies against NOB1 (dilution,

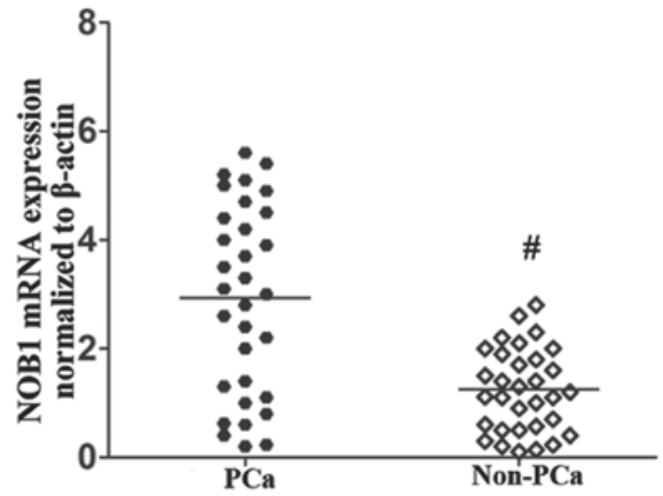

Figure 1. NOB1 mRNA expression is signficantly increased in PCa tissues compared with non-PCa tissues, detected using quantitative polymerase chain reaction analysis. NOB1 expression was normalized to that of $\beta$-actin. Horizontal lines represent the mean. $(n=32)$. ${ }^{~} \mathrm{P}<0.001$ vs. $\mathrm{PCa}$ tissue. $\mathrm{PCa}$, prostate cancer; NOB, nin one binding protein.

1:20; LifeSpan Biosciences Inc.). Sections were then incubated with a HRP rabbit/mouse detection reagent (LifeSpan Biosciences Inc.) for $20 \mathrm{~min}$ at room temperature. Staining was visualized using the diaminobenzidine color substrate. Slides were then counterstained with Mayer's hematoxylin. The absence of primary antibody was used as a negative control.

Immunohistochemical scoring. In order to assess the immunostaining, only nuclear staining was scored. The scoring method was based on the intensity and proportion of positively stained cells. The proportion of positive tumor cells was determined semi-quantitatively and each sample was scored using the following scale: $0,<1 \% ; 1,1-25 \% ; 2,26-50 \%$; $3,51-75 \%$; and 4,76-100\%. The staining intensity was scored as follows: 0 , negative; 1 , weak; 2 , moderate; and 3 , strong. The immunoreactive score of each tumor was calculated as the sum of these two parameters. The immunohistochemical results were finally scored as negative (total score, $0-2$ ) or positive (total score, 3-7). All stained sections were assessed by two independent pathologists without knowledge of the clinicopathological features and any differences in interpretation were resolved by consensus.

Statistical analysis. Statistical analysis was performed using SPSS 17.0 software (SPSS, Inc., Chicago, IL, USA). The $\chi^{2}$ test was used to investigate the significance of the association between NOB1 and the clinicopathological variables. The association between NOB1 expression and prognosis was estimated using univariate and multivariate analyses. Overall survival (OS) and recurrence-free survival (RFS) were analyzed using the Kaplan Meier method followed by log-rank tests. Multivariate analysis was performed using a Cox regression model. $\mathrm{P}<0.01$ was considered to indicate a statistically significant difference.

\section{Results}

NOB1 mRNA expression is increased in PCa tissues. NOB1 mRNA expression was determined using qPCR analysis in 32 fresh PCa samples and paired adjacent non-cancerous 
Table I. Correlation between clinicopathological parameters and NOB1 expression in patients with prostate cancer.

\begin{tabular}{|c|c|c|c|c|}
\hline \multirow[b]{2}{*}{ Parameter } & \multirow[b]{2}{*}{$\begin{array}{c}\text { No. patients } \\
(\%)\end{array}$} & \multicolumn{2}{|c|}{ NOB1 status } & \multirow[b]{2}{*}{$\mathrm{P}$-value } \\
\hline & & $\begin{array}{l}\text { Negative } \\
(n=214)\end{array}$ & $\begin{array}{c}\text { Positive } \\
(n=24)\end{array}$ & \\
\hline Age & & & & 0.861 \\
\hline$<65$ & $220(48)$ & 104 & 116 & \\
\hline$\geq 65$ & $236(52)$ & 110 & 126 & \\
\hline Gleason score & & & & $<0.001$ \\
\hline $2-6$ & $204(45)$ & 112 & 92 & \\
\hline 7 & $228(50)$ & 99 & 129 & \\
\hline $8-10$ & $24(5)$ & 3 & 21 & \\
\hline Distant metastasis & & & & $<0.001$ \\
\hline Absent & $362(79)$ & 204 & 158 & \\
\hline Present & $94(21)$ & 13 & 81 & \\
\hline PSA (ng/ml) & & & & 0.684 \\
\hline$<4$ & $26(6)$ & 16 & 10 & \\
\hline $4-10$ & $192(42)$ & 58 & 134 & \\
\hline$>10$ & $238(52)$ & 140 & 98 & \\
\hline pTNM system & & & & 0.495 \\
\hline $\mathrm{pT} 2 \mathrm{a}, \mathrm{b}, \mathrm{c}$ & $300(66)$ & 136 & 164 & \\
\hline pT3a,b & $156(34)$ & 80 & 76 & \\
\hline
\end{tabular}

Total number of patients $=456$. NOB, nin one binding protein; PSA, prostate-specific antigen; pTNM, pathological tumor-node-metastasis staging.

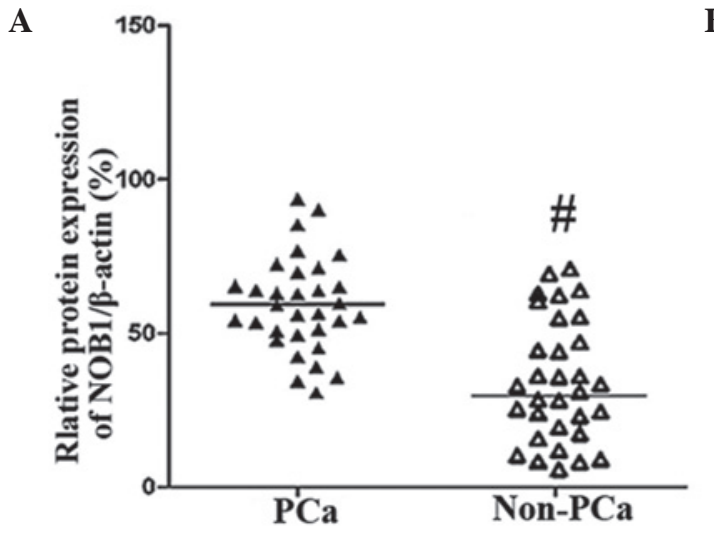

$\mathbf{B}$

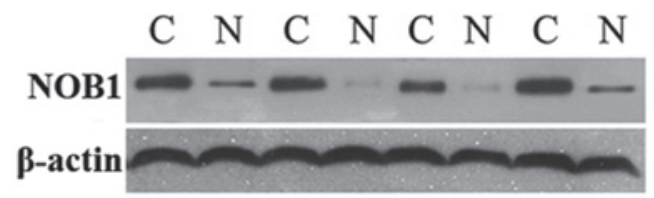

Figure 2. NOB1 protein expression is increased in PCa detected using western blot analysis. (A) Relative NOB1 protein expression in PCa tissues and non-PCa tissues. ${ }^{~} \mathrm{P}<0.001$ vs. PCa tissue ( $\mathrm{n}=32$ ). Horizontal lines represent the mean. (B) Representative western blot of NOB1 protein expression in four paired PCa and matched adjacent non-PCa tissues. C, cancer tissue; N, matched noncancerous tissue; PCa, prostate cancer; NOB, nin one binding protein.

tissues. NOB1 gene expression was found to be significantly increased in the PCa tissues compared with the adjacent non-PCa tissues $(\mathrm{P}<0.001$; Fig. 1).

NOB1 protein expression is increased in PCa tissues. Western blot analysis was performed in the 32 fresh PCa samples and paired adjacent non-cancerous tissues. The protein expression of NOB1 was semiquantified using densitometry. Consistent with the qPCR analysis results, NOB1 protein expression was observed to be significantly increased in the $\mathrm{PCa}$ tissues compared with the matched adjacent non-PCa tissues $(\mathrm{P}<0.001$; Fig. 2).

Correlation between NOB1 expression and clinicopathological parameters in patients with $\mathrm{PCa}$. The mean age of the patients included in the present study was $64.8 \pm 7.9$ years (range, 38-86 years). All patient data and characteristics are summarized in Table I. The immunohistochemical results were graded as negative or positive (Fig. 3). The positive expression rate of NOB1 in PCa samples was 53\% (242/456). 

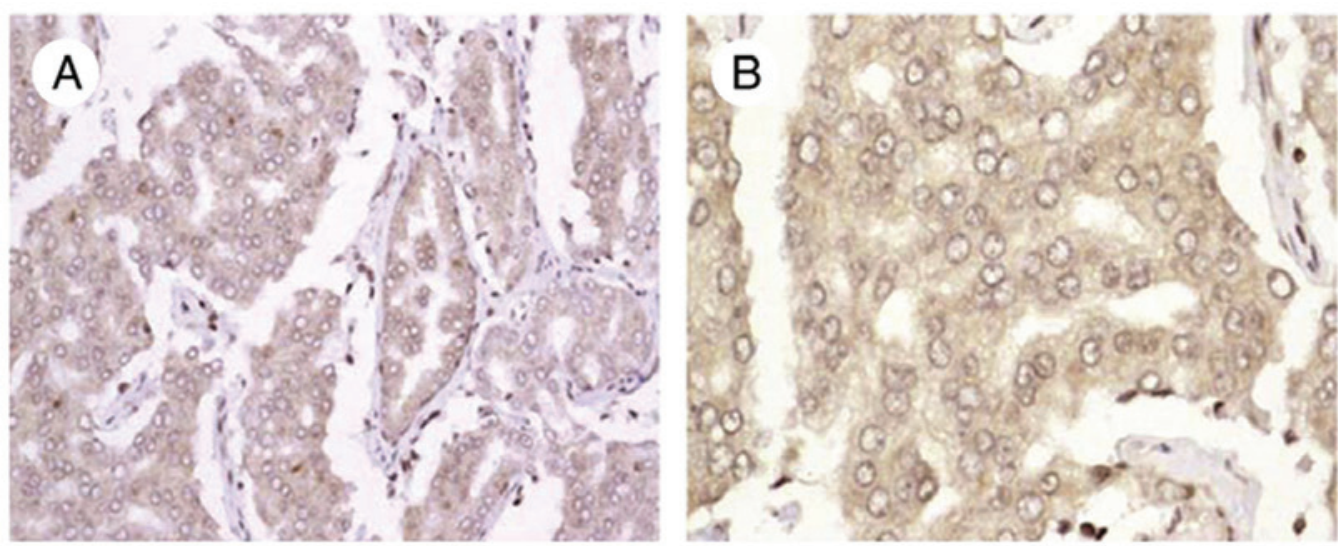

\section{Score 0}
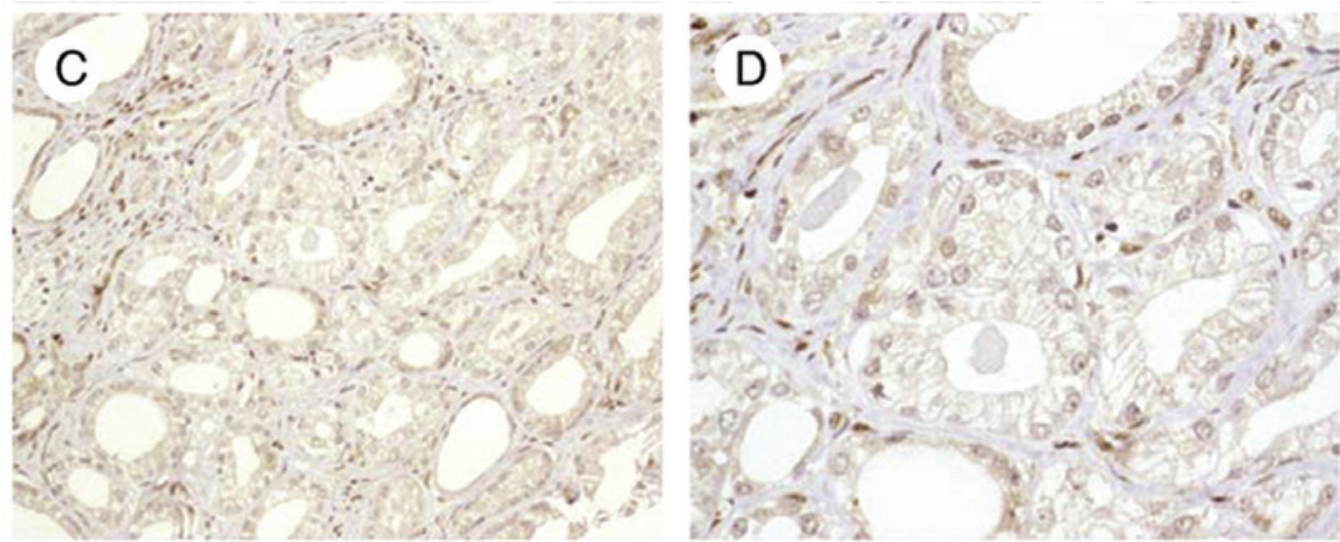

\section{Score 1}
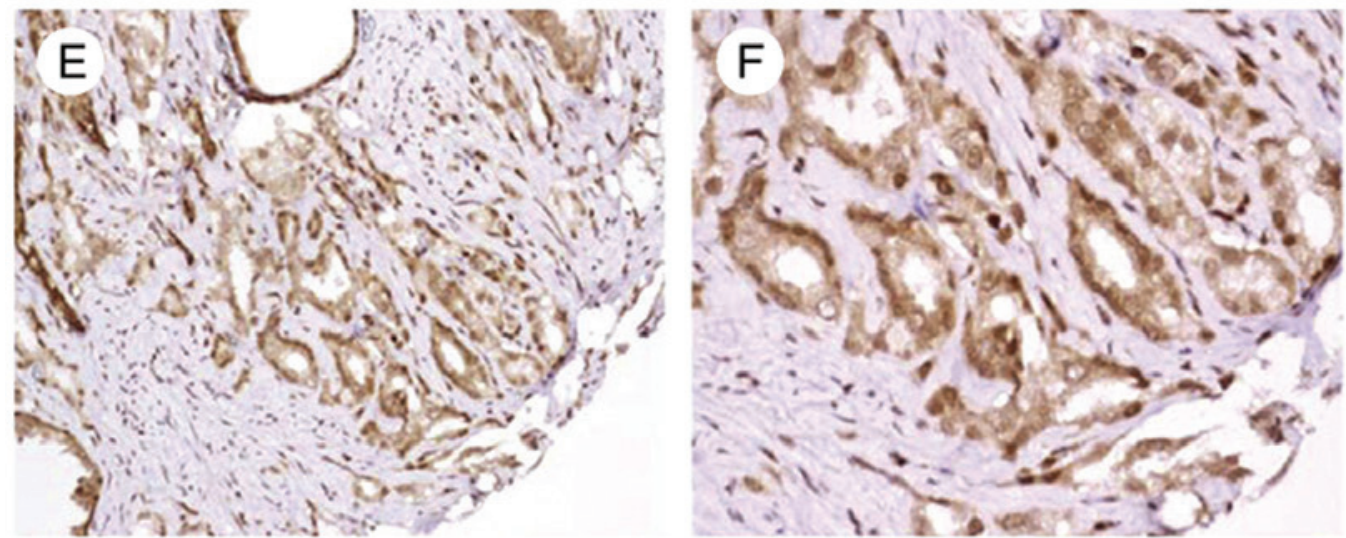

\section{Score 2}
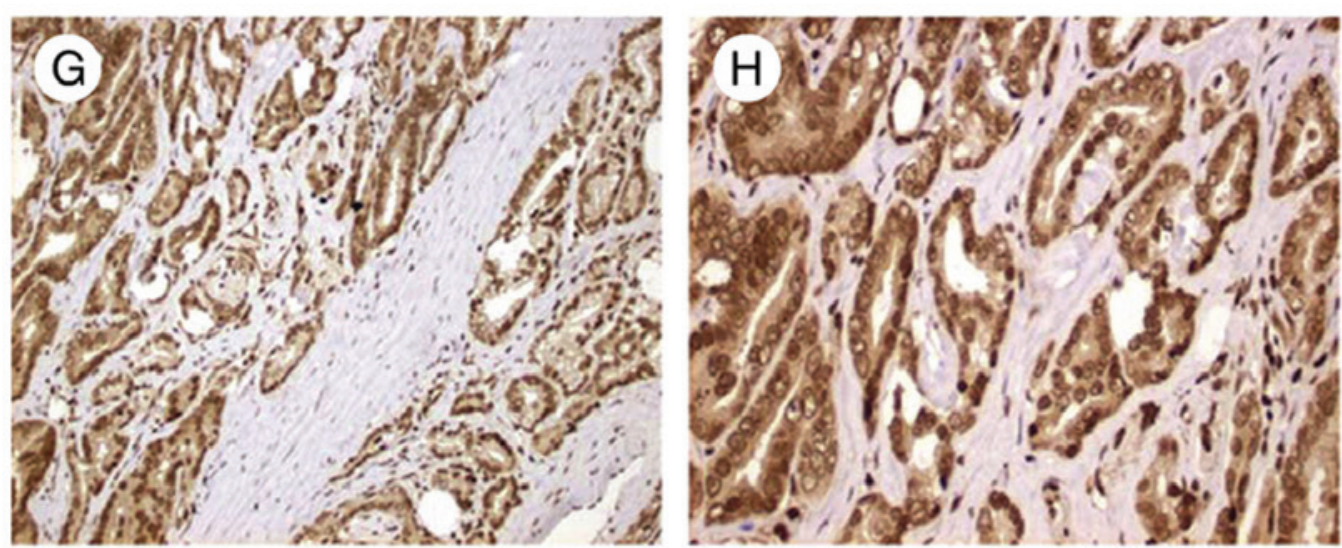

\section{Score 3}

Figure 3. Immunohistochemical detection of NOB1 in PCa. Paraffin-embedded sections of PCa tissue were scored for nuclear NOB1 expression on a scale of 0-3 as follows: 0, negative; 1, weak; 2, moderate; and 3, strong. (A and B) negative staining (magnification x200 and x400, respectively), (C and D) weak staining (magnification, x200 and x400, respectively), (E and F) moderate staining (magnification, x200 and x400, respectively) and (G and H) strong staining (magnification, x200 and x400, respectively). PCa, prostate cancer; NOB, nin one binding protein. 
Table II. Univariate and multivariate analyses of recurrence-free survival in patients with prostate cancer.

\begin{tabular}{|c|c|c|c|c|c|c|}
\hline \multirow[b]{2}{*}{ Parameter } & \multicolumn{3}{|c|}{ Univariate } & \multicolumn{3}{|c|}{ Multivariate } \\
\hline & HR & $95 \% \mathrm{CI}$ & P-value & HR & $95 \% \mathrm{CI}$ & P-value \\
\hline Age ( $\leq 60$ vs. $>60$ years) & 0.86 & $0.50-1.47$ & 0.269 & 0.83 & $0.46-1.58$ & 0.592 \\
\hline Gleason score (2-6 vs. 7 or $8-10)$ & 4.33 & $2.74-5.91$ & $<0.001$ & 5.04 & $3.61-6.18$ & $<0.001$ \\
\hline Distant metastasis (yes vs. no) & 5.48 & $3.67-7.02$ & $<0.001$ & 5.34 & $3.39-7.21$ & $<0.001$ \\
\hline Baseline PSA ( $\leq 10$ vs. $>10$ ng/ml) & 3.74 & $1.89-4.51$ & $<0.001$ & 3.03 & $1.92-5.83$ & $<0.001$ \\
\hline PSA nadir ( $\leq 1$ vs. 1 ng/ml) & 4.21 & $2.97-5.84$ & $<0.001$ & 4.76 & $3.23-6.46$ & $<0.001$ \\
\hline NOB1 (+ vs. -) & 3.45 & $2.34-5.76$ & 0.0012 & 3.54 & $1.41-6.35$ & 0.006 \\
\hline
\end{tabular}

HR, hazard ratio; CI, confidence interval; PSA, prostate-specific antigen; NOB, nin one binding protein.

A

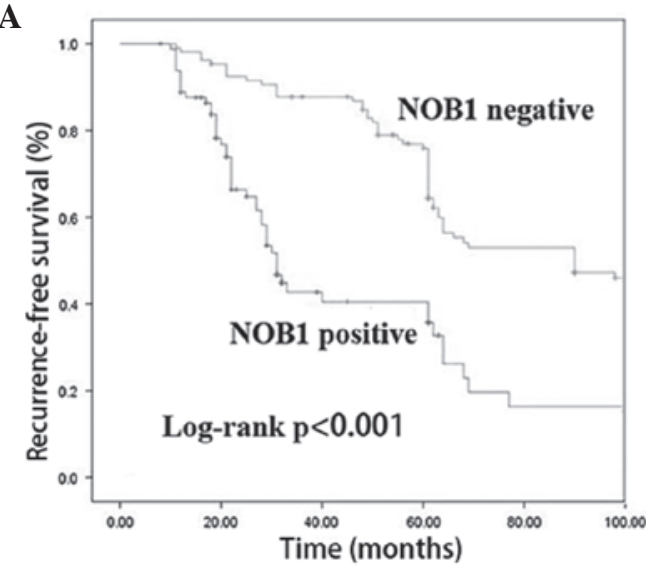

B

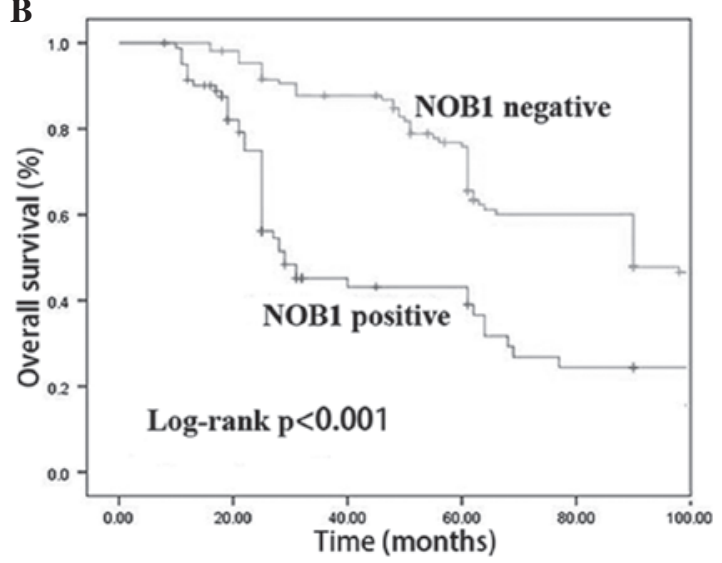

Figure 4. (A) Recurrence-free survival and (B) overall survival curves based on NOB1 expression in patients with prostate cancer. NOB, nin one binding protein.

By contrast, only $6.1 \%$ (28/456) of the paired adjacent non-PCa tissues showed positive NOB1 expression. As shown in Table I, positive NOB1 expression was significantly correlated with the Gleason score $(\mathrm{P}<0.001)$ and distant metastasis $(\mathrm{P}<0.001)$.

Correlation between positive NOB1 expression and clinical outcome. The mean follow-up period was 39.6 months (range, 6-92 months). Among the 456 patients with $\mathrm{PCa}, 12$ (2.6\%) were lost during the follow-up period and 68 (14.9\%) succumbed to their disease. During the follow-up period, the RFS of the patients with positive NOB1 expression was observed to be significantly decreased, as compared with that of the patients with negative NOB1 expression $(\mathrm{P}<0.001)$. The OS rate of the patients with positive NOB1 expression was also significantly lower compared with that of the patients with negative NOB1 expression $(\mathrm{P}<0.001$; Fig. 4).

Univariate analysis (Table II) revealed that higher Gleason score, higher prostate-specific antigen nadir, positive distant metastasis and positive NOB1 expression were correlated with lower RFS. Compared with the patients with positive NOB1 expression, the patients with negative NOB1 expression had significantly enhanced clinical outcomes in terms of RFS $(\mathrm{P}<0.001)$. Multivariate analysis revealed that
NOB1 expression was a significant predictor of RFS, independent of other clinicopathological variables (hazard ratio, $3.45 ; 95 \%$ confidence interval, 1.41-6.35; $\mathrm{P}=0.006)$.

\section{Discussion}

Identification of PCa-specific biomarkers is important for diagnosis, therapy and prognostic prediction. The present study investigated the expression of NOB1 in 456 patients with $\mathrm{PCa}$ and its association with patient survival and clinicopathological characteristics. The findings suggest that positive NOB1 expression was present in $53 \%$ of PCa cases. Furthermore, significant correlation was observed between positive NOB1 expression and poor prognosis, independent of other patient characteristics. These results indicate that NOB1 expression may be a novel prognostic marker for $\mathrm{PCa}$.

The NOB1 gene was identified to be an oncogene responsible for the high proliferation rates found in cancer cells (11). Several studies have reported that the repression of NOB1 gene expression inhibits the growth of ovarian cancer and glioma $(11,12)$. Downregulation of NOB1 has been found to inhibit the growth of human hepatocellular carcinoma cells (13). Furthermore, small interfering RNA-mediated silencing of NOB1 inhibits the proliferation and growth of breast cancer cells (14). Previous studies have also associated the expression of NOB1 with 
clinical outcome in papillary thyroid carcinoma and breast infiltrating ductal carcinoma $(15,16)$. Thus, the present study investigated NOB1 mRNA expression in PCa samples using qPCR analysis and NOB1 protein expression in primary PCa tissues using western blot analysis. Results revealed that NOB1 mRNA and protein levels were significantly increased in tumor tissue samples compared with those in the adjacent non-tumor tissue samples. These results suggest that NOB1 may have a role in PCa.

In the present study, NOB1 protein expression was analyzed in tumor tissue in order to assess its prognostic significance for PCa. Immunohistochemical analysis demonstrated high NOB1 expression in 53\% (242/456) of the patients with PCa. Moreover, in a relatively large population of patients with $\mathrm{PCa}(\mathrm{n}=456)$, high expression of NOB1 was found to be significantly correlated with the Gleason score and distant metastasis of $\mathrm{PCa}$, suggesting that increased NOB1 expression may promote tumor growth and invasion. These results suggest that NOB1 may have an important role in the tumorigenesis and progression of $\mathrm{PCa}$.

Kaplan-Meier survival analysis revealed that patients with positive NOB1 expression had a significantly reduced OS and RFS compared with the patients with negative NOB1 expression. Univariate analyses showed that increased NOB1 expression in $\mathrm{PCa}$ tissues was significantly correlated with RFS. Furthermore, Cox hazard ratio regression analyses demonstrated that NOB1 expression was an independent prognostic factor of disease free survival in patients with PCa. These results suggest that NOB1 may be an important prognostic marker for patients with PCa.

In conclusion, the present study has demonstrated that NOB1 expression in PCa is correlated with a more malignant phenotype and worse clinical outcome in a relatively large number of PCa samples. These findings suggest that NOB1 may function as a potential prognostic indicator for $\mathrm{PCa}$. Translational and prospective studies of NOB1 as a therapeutic target in $\mathrm{PCa}$ are required.

\section{Acknowledgements}

The authors would like to thank the Innovation Program of the Shanghai Municipal Education Commission for grant funding awarded to Dr Xingang Cui (grant no.14zz084).

\section{References}

1. Jemal A, Siegel R, Xu J and Ward E: Cancer statistics, 2010. CA Cancer J Clin 60: 277-300, 2010

2. Kim WT and Kim WJ: MicroRNAs in prostate cancer. Prostate Int 1: 3-9, 2013.

3. Madan RA and Arlen PM: Recent advances revolutionize treatment of metastatic prostate cancer. Future Oncol 9: 1133-1144, 2013.

4. Facompre N and El-Bayoumy K: Potential stages for prostate cancer prevention with selenium: implications for cancer survivors. Cancer Res 69: 2699-2703, 2009.

5. Zhang Y, Ni J,Zhou G, Yuan J, Ren W, Shan Y, Tang W, Yu L and Zhao S: Cloning, expression and characterization of the human NOB1 gene. Mol Biol Rep 32: 185-189, 2005.

6. Lamanna AC and Karbstein K: Nob1 binds the single-stranded cleavage site D at the 3'-end of 18S rRNA with its PIN domain. Proc Natl Acad Sci USA 106: 14259-14264, 2009.

7. Hong L, Piao Y, Han Y, Wang J, Zhang X, Du Y, Cao S, Qiao T, Chen Z and Fan D: Zinc ribbon domain-containing 1 (ZNRD1) mediates multidrug resistance of leukemia cells through regulation of P-glycoprotein and Bcl-2. Mol Cancer Ther 4: 1936-1942, 2005.

8. Granneman S, Nandineni MR and Baserga SJ: The putative NTPase Fap7 mediates cytoplasmic 20S pre-rRNA processing through a direct interaction with Rps14. Mol Cell Biol 25: 10352-10364, 2005.

9. Wang H, Li P and Zhao B: Knockdown of NOB1 expression by RNAi inhibits cellular proliferation and migration in human gliomas. Gene 528: 146-153, 2013.

10. D'Amico AV, Moul JW, Carroll PR, Sun L, Lubeck D and Chen MH: Surrogate end point for prostate cancer-specific mortality after radical prostatectomy or radiation therapy. J Natl Cancer Inst 95: 1376-1383, 2003.

11. Lin Y, Peng S, Yu H, Teng H and Cui M: RNAi-mediated downregulation of NOB1 suppresses the growth and colony-formation ability of human ovarian cancer cells. Med Oncol 29: 311-317, 2012.

12. Zhou J, Xu T, Yan Y, Qin R, Wang H, Zhang X, Huang Y, Wang Y, Lu Y, Fu D and Chen J: MicroRNA-326 functions as a tumor suppressor in glioma by targeting the Nin one binding protein (NOB1). PLoS One 8: e68469, 2013.

13. Lu Z, Guo Q, Shi A, Xie F and Lu Q: Downregulation of NIN/RPN12 binding protein inhibit the growth of human hepatocellular carcinoma cells. Mol Biol Rep 39: 501-507, 2012.

14. Huang WY, Chen DH, Ning L and Wang LW: siRNA mediated silencing of NIN1/RPN12 binding protein 1 homolog inhibits proliferation and growth of breast cancer cells. Asian Pac J Cancer Prev 13: 1823-1827, 2012.

15. Lin S, Meng W, Zhang W, Liu J, Wang P, Xue S and Chen G: Expression of the NOB1 gene and its clinical significance in papillary thyroid carcinoma. J Int Med Res 41: 568-572, 2013.

16. Li XY, Luo QF, Li J, Wei CK, Kong XJ, Zhang JF and Fang L: Clinical significance of NOB1 expression in breast infiltrating ductal carcinoma. Int J Clin Exp Pathol 6: 2137-2144, 2013. 